\title{
CTBot: A Stereotactic-Guided Robotic Assistant for Percutaneous Procedures of the Abdomen
}

\author{
Benjamin Maurin ${ }^{1}$, Christophe Doignon ${ }^{1}$, Jacques Gangloff ${ }^{1}$, Bernard Bayle ${ }^{1}$, \\ Michel de Mathelin ${ }^{1}$, Olivier Piccin ${ }^{2}$, Afshin Gangi ${ }^{3}$ \\ ${ }^{1}$ LSIIT, Strasbourg I University, Bd. S. Brant, 67412 Illkirch, FRANCE; \\ ${ }^{2}$ LICIA(EA3434), INSA-Strasbourg, 24 Bd de la Victoire, 67084 Strasbourg, FRANCE; \\ 3 Department of Radiology B, University Hospital of Strasbourg, 67091 Strasbourg, FRANCE
}

\begin{abstract}
This article presents positioning results of a stereotactic robotic assistant for percutaneous needle insertions in the abdomen. The robotic system, called the CT-Bot, is succinctly described. This mechanically safe device is compatible with medical requirements and offers a novel approach to robotic needle insertion with computed tomography guidance. Our system does self-registration using only visual information from a fiducial marker. The theoretical developments explain how the pose reconstruction is done using only four fiducial points and how the automatic registration algorithm is achieved. The results concern the automatic positioning of the tip of a needle with respect to a reference point selected in a CT-image. The accuracy of the positioning result show how interesting this system is for clinical use.
\end{abstract}

Keywords: CT-guided robotic assistant, robotized percutaneous procedures, stereotactic registration

\section{INTRODUCTION}

For diagnosis or therapy, radiologists often use Computed Tomography scanners (CT-scans) to accurately insert needles in internal organs of the abdomen. During highly precise interventions, about $1 \mathrm{~mm}$ of accuracy, computed tomography has proved to be an excellent imaging modality given its resolution and the good tissue differentiation. These procedures are not totally safe for the radiologist who is exposed to high amount of X-rays during CT-guided needle insertions. Such interventions are potentially harmful when performing a large number of them.

Consequently, given the accuracy needs as well as the necessary X-rays protection, more and more visuallyguided robotic systems for percutaneous procedures have been proposed in the literature (see Taylor ${ }^{1}$ for a good presentation). Even if clinical trials have already been achieved in some cases, current systems are not well suited for abdominal interventions where the motions and the respiration of the patient create large disturbances that are difficult to compensate with a static needle holder.

We recently proposed some novels ideas that we used in the design of a new robotic system using a parallel mechanism. ${ }^{2}$ The main novelty was that our robotic assistant involves two different kind of motions: first the positioning and orientation of a line supporting the needle using a parallel structure and second a needle driver unit that inserts the needle and spins it about its axis. This allows for security and safety of the patient while authorizing full movement of the needle and force measurement. Since this system is part of a complete project that start from the pre-operative images of a patient to the real intervention in the medical operating room, an important point is the registration of the robot with respect to the patient.

Starting from the workflow of the intervention and the robotic setup, this paper describes the registration method that allows to reconstruct the robot position in the CT-scan image with only 4 points. Then an automatic matching algorithm is proposed in order to solve the correspondence issue without human intervention. These methods are used to place the tip of a needle to a given target in the CT-image space with the robotic assistant. A needle positioning result is given, that shows a point-and-click relative displacement computed with the registration algorithm. We conclude on the possible benefits the overall system will offer to radiologists.

Further author information: E-mail maurin@eavr.u-strasbg.fr 


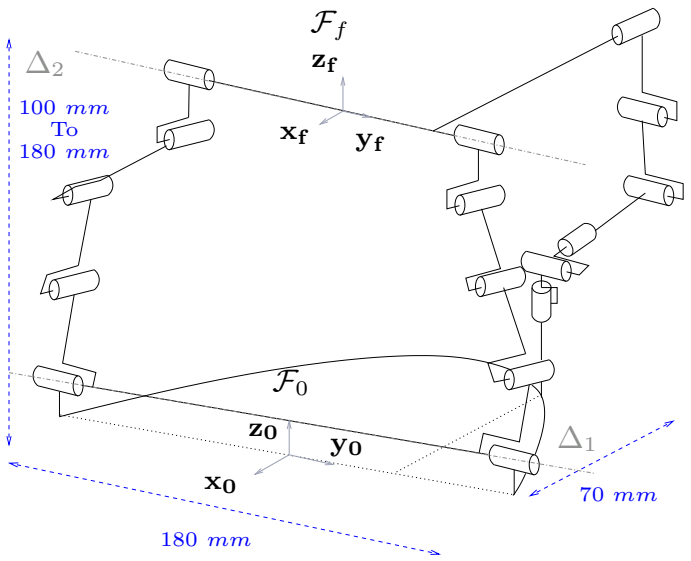

(a)

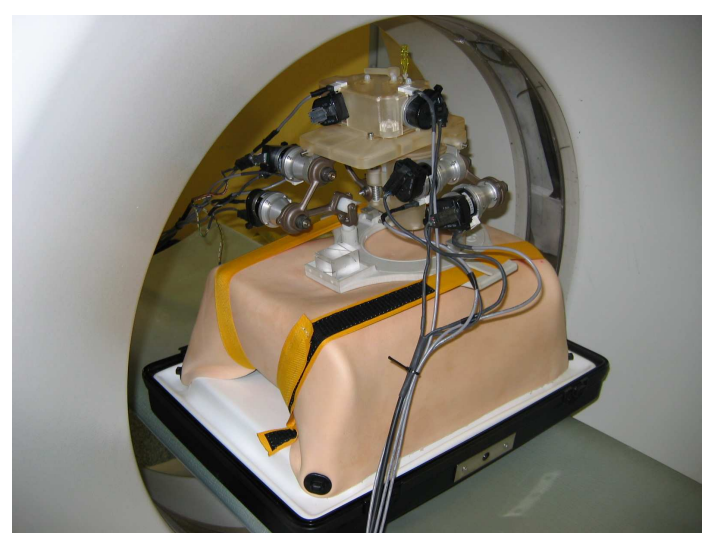

(b)

Figure 1. (a) The kinematics of the robotic device. - (b) The robotic system placed on an abdominal phantom, inside the CT-scan tunnel.

\section{THE CT-BOT ROBOTIC ASSISTANT}

\subsection{Short Description of the Robotic System}

The system is made up of a parallel robotic positioning device, a control unit (standard pc with power amplifiers) and a supervising computer that does the image processing. A graphical user interface is also running on the supervising computer.

The prototype is placed on the abdomen of the patient to compensate annoying motions like natural breathing. It fits in a $20 \mathrm{~cm}^{3}$ cube and its weight is about $3 \mathrm{~kg}$. Only few metallic parts are crossing the X-rays plane to ensure the CT-scan compatibility. The CT-bot device is presented in previous works ${ }^{2,3}$ where the authors also explain the kinematics (closed-form) and the design constraints. The main novelty was the use of a light and compact positioner with five degrees of freedom that has an almost spherical workspace (see Fig. 1.(a)). This positioner is powered with piezo-electrical motors and is intrinsically safe: if a critical failure occurs, the system stops with no motion.

A picture representing the experimental setup used during the experiments is shown in Fig. 1.(b). Currently, the system is attached on the abdomen using straps, which has some inconvenient concerning the comfort of the patient and the stability of the base. This issue will be solved in a futur design.

\subsection{Robotized Percutaneous Procedure of the Abdomen}

The procedure of a typical robotic insertion is decomposed in different steps (presented on Fig. 2.(a)). This workflow is very close to the one the radiologist does in manual interventions. This paper focuses on the steps during the beginning of the intervention, when the radiologist selects her/his trajectory in the CT images. In manual interventions, these planning steps take a major part of the total time (about the quarter), and reducing this time can be valuable for both the practitioner and the patient. The difficulty for the radiologist is often the positioning of the tip of the needle on the entry point, without being exposed to high amount of X-rays.

In contrast, the major difficulty that the robotic approach is facing is the registration of the end-effector holding the needle in the CT-image. Many approach are exploited, using either external devices like Optical Trackers ${ }^{4}$ or CT-visible fiducial objects (like a classical BRW stereotactic frame ${ }^{5}$ ). The first approach also requires the registration of the optical system with respect to the image frame to achieve a correct image-guided procedure. 


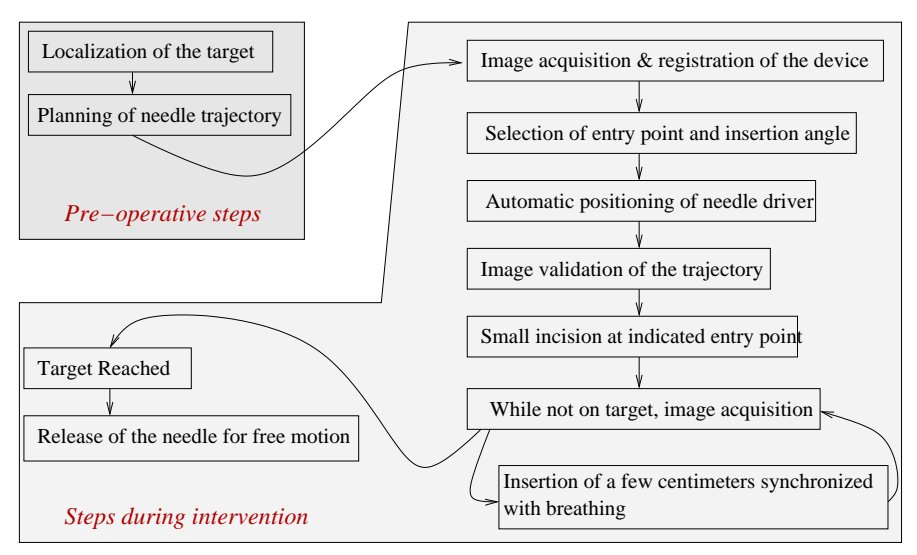

(a)

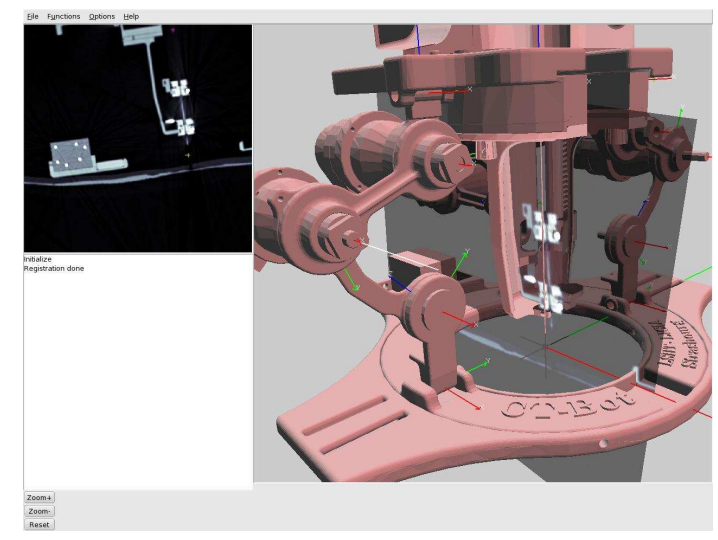

(b)

Figure 2. (a) Workflow of a robotized percutaneous intervention. - (b) The graphical user interface build to control the robotic device and the registration process.

In our case, the second method was chosen since it is an easier way to compute the trajectory of the needle in the image when the robotic manipulator is well registered in the image (eye to hand configuration). When a DICOM image is send to the supervising computer, the image is displayed in the graphical user interface and the registration process is done. Without human intervention, the robot is automatically registered with respect to the image. Then, the radiologist can choose the best entry point and the orientation of the needle.

Currently, the insertion step is made manualy and the robotic device only constraints the direction of the needle, without interfering with the CT-scan.

\section{SINGLE SLICE POSE RECONSTRUCTION WITH FOUR MATCHED CORRESPONDENCES}

The registration problem with a single CT slice is also studied by Susil $^{6}$ et $A l$. were the authors proposed a geometric framework, based on distance relationships. Also Lee ${ }^{7}$ et Al. used a similar approach to numericaly find the correct pose. But both methods require a high number of fiducial points without an automatic registration solution.

\subsection{Geometric modeling of the CT-scan}

The CT-scan is considered as a vision sensor which gives tomographic images of the fiducial object. The registration issue is then solved using a general geometric framework rather than analyzing the physical properties of the tomographic reconstruction. The imaging model we present has been partly studied in another work. ${ }^{8}$ This geometric model involves an affine transformation (with anisotropic scalings) accounting for intrinsic parameters, an euclidean one for the rigid-body transformation and an orthographic projection. To formulate the registration problem, also called the pose reconstruction problem in our case, we define a few reference coordinate frames: $\mathcal{F}_{0}$ for the object, $\mathcal{F}_{c t}$ for the CT slice, and $\mathcal{F}_{I}$ for the CT image with pixel units instead of millimeters.

To model the fiducial object, we name the constituent lines as $\Delta_{i}$ for the $i^{\text {th }}$ object line. When this line intersects the cutting plane $(\pi)$, the intersection is a point $\mathbf{P}_{i}$ in the $3 \mathrm{D}$ space that belongs to the CT image (see Fig. 3(a)). An euclidean transformation between the object reference frame $\mathcal{F}_{0}$ attached to the set of rigid straight lines and the CT-scan reference frame $\mathcal{F}_{c t}$ is

$$
{ }^{0} \mathbf{O P} \mathbf{P}_{i}={ }^{0} R_{c t}{ }^{c t} \mathbf{O} \mathbf{P}_{i}+{ }^{0} \mathbf{t}
$$




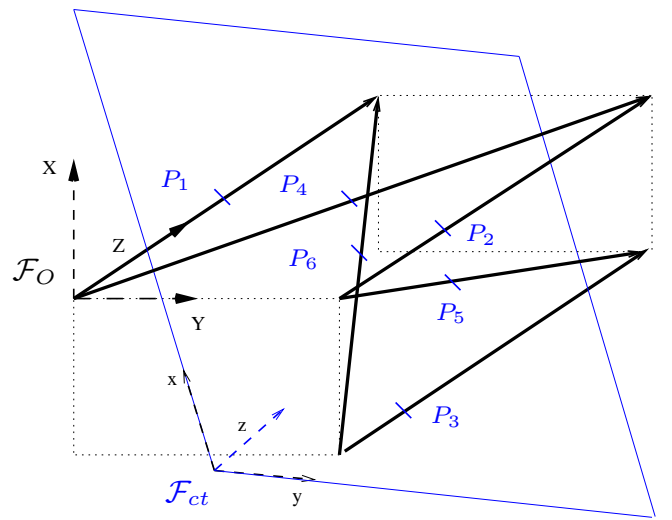

(a)

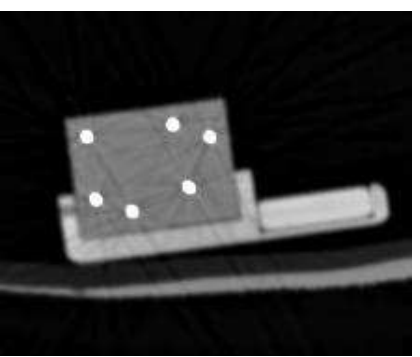

(b)

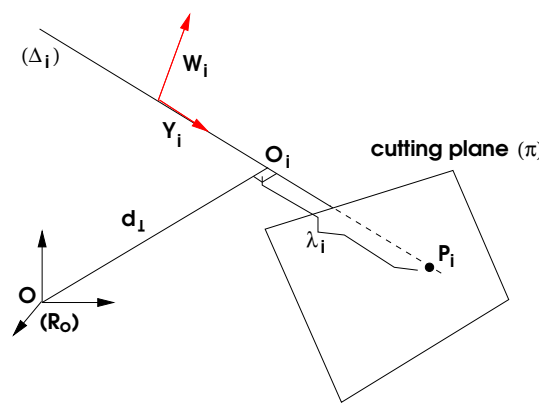

(c)

Figure 3. (a) Fiducial lines are crossing the cutting plane at points $Q_{i}$. - (b) The CT-scan image obtained with the fiducial object. - (c)A 3-D line $\Delta_{i}$ crossing the cutting plane $(\pi)$. The pair of vectors $\left(\mathbf{y}_{i}, \mathbf{w}_{i}\right)$ is the Plückerian representation of the line.

where the orthonormal matrix ${ }^{0} R_{c t}$ and the vector ${ }^{0} \mathbf{t}$ are the parameters of a rigid-body transformation. Also, the image is made of pixels, that means a scaling transformation occurs between a point in $\mathcal{F}_{c t}$ and the projected point in the image with pixel units:

$$
{ }^{c t} \mathbf{O P}_{i}=\left[\begin{array}{c}
x_{i} \\
y_{i} \\
z_{i}
\end{array}\right]=\underbrace{\left[\begin{array}{ll}
1 & 0 \\
0 & 1 \\
0 & 0
\end{array}\right]}_{\text {orthographic }} \underbrace{\left[\begin{array}{cc}
s_{x} & 0 \\
0 & s_{y}
\end{array}\right]}_{\text {intrinsic parameters }} \underbrace{\left[\begin{array}{c}
u_{i} \\
v_{i}
\end{array}\right]}_{\text {pixel }}={ }^{c t} \Pi_{\pi}{ }^{\pi} S_{I}{ }^{I} \mathbf{Q}_{i}
$$

Hence, the imaging model maps a point ${ }^{c t} \mathbf{O P}_{i}=\left(x_{i}, y_{i}, z_{i}\right)^{T}$ in the CT-scan reference frame to an image coordinates ${ }^{I} \mathbf{Q}_{i}=\left(u_{i}, v_{i}\right)^{T}$ in the image frame $\mathcal{F}_{I}$. In the last equation, ${ }^{c t} \Pi_{\pi}$ is a $(3 \times 2)$ matrix accounting for the orthographic projection onto the image (cutting plane $(\pi)$ ) in the canonical form and the $2 \times 2$ matrix ${ }^{\pi} S_{I}$ is the scaling transformation accounting for the size of a pixel. These values are the intrinsic parameters of the scanner (positive scaling factors $s_{x}$ and $s_{y}$ ), and are supposed to be known either by a previous calibration or by the values given by the CT-scan itself. Combining Eq. (1) and (2) leads to

$$
{ }^{0} \mathbf{O P}_{i}=\left[\begin{array}{cc}
{ }^{0} \mathbf{r}_{1} & { }^{0} \mathbf{r}_{2}
\end{array}\right]{ }^{\pi} S_{I}\left[\begin{array}{c}
u_{i} \\
v_{i}
\end{array}\right]+{ }^{0} \mathbf{t}
$$

which is the compact representation of the transformation $\mathcal{F}_{I} \rightarrow \mathcal{F}_{0}$, also called the imaging model. The component ${ }^{0} \mathbf{r}_{j}$ is the $j^{\text {th }}$ column of the rotation matrix ${ }^{0} R_{c t}$ which satisfy the quadratic constraints emanating from the orthonormality of any rotation matrix:

$$
{ }^{0} \mathbf{r}_{1}{ }^{T}{ }^{0} \mathbf{r}_{1}=1, \quad{ }^{0} \mathbf{r}_{2}{ }^{T}{ }^{0} \mathbf{r}_{2}=1, \text { and }{ }^{0} \mathbf{r}_{1}{ }^{T}{ }^{0} \mathbf{r}_{2}=0 .
$$

and the product $\left(\left[{ }^{0} \mathbf{r}_{1}{ }^{0} \mathbf{r}_{2}\right]{ }^{\pi} S_{I}\right)$ is a $(3 \times 2)$ real matrix.

\subsection{Modeling of the fiducial object}

The main additions to our previous work ${ }^{8}$ are the way the fiducial object is modeled, and the number of fiducial points needed to solve the registration problem. As for stereotactic frames, ${ }^{9}$ the fiducial object is made of straight lines. This geometric element is very well suited for tomography since it is easily modeled and the cross section with the cutting plane is a point. 
The $i^{\text {th }}$ object line $\Delta_{i}$ may be represented by an origin $\mathbf{O}_{i}(3$ dof $)$ and a unit direction vector $\mathbf{y}_{i}(2$ dof $)$ as shown on Fig. 3.(c)). This is an overconstrained parametric representation since any line is defined with only 4 dof in the 3-D space. $\mathbf{O}_{i}$ is chosen as the orthogonal projection of the origin of the frame $\mathcal{F}_{O}$ onto $\Delta_{i}$ that also verify $\mathbf{O}_{i} \mathbf{P}_{i} \times \mathbf{y}_{i}=\mathbf{0}$. Hence a point $\mathbf{P}_{i}$ that belongs to $\Delta_{i}$, is expressed as

$$
{ }^{0} \mathbf{O P}_{i}={ }^{0} \mathbf{O O}_{i}+\lambda_{i}{ }^{0} \mathbf{y}_{i} \quad, \quad \lambda_{i} \in \mathbb{R} .
$$

Therefore, using Eq. (3), one must solve for $\mathbf{r}_{1}, \mathbf{r}_{2}, \mathbf{t}$ and $\left\{\lambda_{i}\right\}$ using:

$$
{ }^{0} \mathbf{O O}_{i}+\lambda{ }_{i}{ }^{0} \mathbf{y}_{i}=\left[\begin{array}{cc}
{ }^{0} \mathbf{r}_{1} & { }^{0} \mathbf{r}_{2}
\end{array}\right]{ }^{\pi} S_{I}\left[\begin{array}{c}
u_{i} \\
v_{i}
\end{array}\right]+{ }^{0} \mathbf{t} .
$$

This latter equation can be linearly solved using a least-square method or a Newton-like optimization with at least 5 points (each point provides 3 linear equations, but also the unknown $\left\{\lambda_{i}\right\}^{8}$ ). To remove the $\left\{\lambda_{i}\right\}$ dependencies, we introduce the Plückerian representation for a 3 -D line $\Delta_{i}$ (see Hartley \& Zisserman ${ }^{10}$ for instance). With this representation, the explicit definition of the origin of the line is removed. Using the previous vector $\mathbf{y}_{i}$ and a vector $\mathbf{w}_{i}$ defined as $\mathbf{w}_{i}=\mathbf{y}_{i} \times \mathbf{O P}_{i}$, the Plückerian coordinates $\left(\mathbf{y}_{i}, \mathbf{w}_{i}\right)$ of a line $\Delta_{i}$ are such that:

$$
\begin{aligned}
\mathbf{y}_{i} & =\mathbf{O}_{i} \mathbf{P}_{i} /\left\|\mathbf{O}_{i} \mathbf{P}_{i}\right\| \\
\mathbf{w}_{i} & =\mathbf{y}_{i} \times \mathbf{O P}_{i}=\mathbf{y}_{i} \times \mathbf{O O}_{i} \Rightarrow{ }^{0} \mathbf{w}_{i}^{T}{ }^{0} \mathbf{O} \mathbf{P}_{i}=0 .
\end{aligned}
$$

$\left\|\mathbf{w}_{i}\right\|=\left\|\mathbf{O O}_{i}\right\|$ is the orthogonal distance $d_{\perp}$ from the line to the origin. The above definition for Plückerian object line coordinates $\left(\mathbf{y}_{i}, \mathbf{w}_{i}\right)$ and the latter expression of ${ }^{0} \mathbf{O} \mathbf{P}_{i}$ can be gathered in the inhomogeneous equation:

$$
{ }^{0} \mathbf{w}_{i}=\left[{ }^{0} \mathbf{y}_{i}\right]_{\times}\left(\left[\begin{array}{cc}
{ }^{0} \mathbf{r}_{1} & { }^{0} \mathbf{r}_{2}
\end{array}\right]{ }^{\pi} S_{I}\left[\begin{array}{c}
u_{i} \\
v_{i}
\end{array}\right]+{ }^{0} \mathbf{t}\right)
$$

Equation (8) is the basis of our registration approach. In practice, the lines of the fiducial object are straight rods and are bounded in length $\left(\lambda^{\text {min }} \leq \lambda \leq \lambda^{\text {max }}\right)$. It is very relevant to compute these extremal values for any displacement $\left({ }^{0} R_{c t},{ }^{0} \mathbf{t}\right)$ to check for a real intersection with the cutting plane, that is to verify the visibility of a spot in the cross sectional image and therefore the relevance of that spot. This is a key feature to better control the displacement. To do so, the previous expression for vector $\mathbf{O O}_{i}$ in Eq.(6) is developed and a pre-multiplication with ${ }^{0} \mathbf{y}_{i}^{T}$ leads to

$$
{ }^{0} \mathbf{y}_{i}^{T}{ }^{0} \mathbf{O} \mathbf{O}_{i}+\lambda{ }_{i}{ }^{0} \mathbf{y}_{i}^{T}{ }^{0} \mathbf{y}_{i}={ }^{0} \mathbf{y}_{i}{ }^{T}\left(\left[{ }^{0} \mathbf{r}_{1}{ }^{0} \mathbf{r}_{2}\right]{ }^{\pi} S_{I}\left[\begin{array}{c}
u_{i} \\
v_{i}
\end{array}\right]+{ }^{0} \mathbf{t}\right)
$$

Due to Plückerian coordinates, one has that ${ }^{0} \mathbf{y}_{i}^{T}{ }^{0} \mathbf{y}_{i}=1$ and $\mathbf{y}_{i}$ is orthogonal to $\mathbf{O O}_{i}$. The final expression for the scalar $\lambda_{i}$ (which does not depend on vector $\mathbf{w}_{i}$ ) is

$$
\lambda_{i}={ }^{0} \mathbf{y}_{i}{ }^{T}\left(\left[{ }^{0} \mathbf{r}_{1}{ }^{0} \mathbf{r}_{2}\right]{ }^{\pi} S_{I}\left[\begin{array}{l}
u_{i} \\
v_{i}
\end{array}\right]+{ }^{0} \mathbf{t}\right) .
$$

\subsection{The pose reconstruction problem}

Once an image of the fiducial is obtained and a small number of correspondences between image points and fiducial lines is provided, one has to solve a linear system. The set of equations can be written as

$$
\begin{aligned}
& { }^{0} \mathbf{w}_{i}=\left[{ }^{0} \mathbf{y}_{i}\right]_{\times}\left(\left[\begin{array}{ll}
{ }^{0} \mathbf{r}_{1} & { }^{0} \mathbf{r}_{2}
\end{array}\right]{ }^{\pi} S_{I}\left[\begin{array}{c}
u_{i} \\
v_{i}
\end{array}\right]+{ }^{0} \mathbf{t}\right) \\
& =\left[{ }^{0} \mathbf{y}_{i}\right]_{\times}\left(u_{i} s_{x}{ }^{0} \mathbf{r}_{1}+v_{i} s_{y}{ }^{0} \mathbf{r}_{2}+{ }^{0} \mathbf{t}\right) \\
& =\left[{ }^{0} \mathbf{y}_{i}\right]_{\times}\left[\begin{array}{lllll}
u_{i} s_{x} I_{3} & v_{i} s_{y} I_{3} & I_{3}
\end{array}\right]\left[\begin{array}{c}
{ }^{0} \mathbf{r}_{1} \\
{ }^{0} \mathbf{r}_{2} \\
{ }^{0} \mathbf{t}
\end{array}\right] \\
& =\left[\left(u_{i} s_{x}, v_{i} s_{y}, 1\right) \otimes\left[{ }^{0} \mathbf{y}_{i}\right]_{\times}\right]\left[\begin{array}{c}
{ }^{0} \mathbf{r}_{1} \\
{ }^{0} \mathbf{r}_{2} \\
{ }^{0} \mathbf{t}
\end{array}\right]
\end{aligned}
$$


with $I_{3}$ the $(3 \times 3)$ identity matrix and $\otimes$ the matrix kronecker product. Since $R$ is orthonormal, one has the following problem to solve:

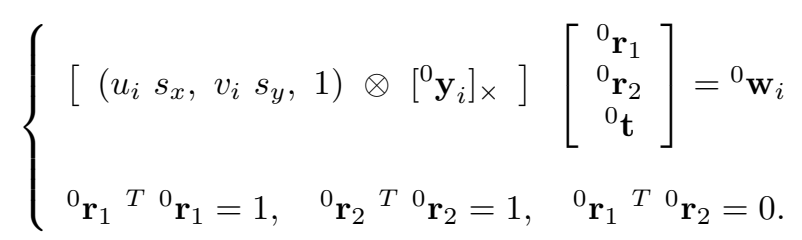

By supposing that $n$ lines are in relation with $n$ points, one can rewrite (12) as an optimization problem with equality constraint:

$$
\left\{\begin{array}{c}
\min _{\mathbf{x}}\|A \mathbf{x}-\mathbf{b}\|^{2} \\
\mathbf{x}^{T} C_{1} \mathbf{x}=1, \quad \mathbf{x}^{T} C_{2} \mathbf{x}=1, \quad \mathbf{x}^{T} C_{3} \mathbf{x}=0
\end{array}\right.
$$

with all the unknown stacked in a $(9 \times 1)$ vector $\mathbf{x}$ and constraint matrices $C_{1}, C_{2}, C_{3}$ such that

$$
\mathbf{x}=\left[\begin{array}{c}
{ }^{0} \mathbf{r}_{1} \\
{ }^{0} \mathbf{r}_{2} \\
{ }^{0} \mathbf{t}
\end{array}\right], \quad C_{1}=\left[\begin{array}{ccc}
I_{3} & 0_{3} & 0_{3} \\
0_{3} & 0_{3} & 0_{3} \\
0_{3} & 0_{3} & 0_{3}
\end{array}\right], \quad C_{2}=\left[\begin{array}{ccc}
0_{3} & 0_{3} & 0_{3} \\
0_{3} & I_{3} & 0_{3} \\
0_{3} & 0_{3} & 0_{3}
\end{array}\right], \quad C_{3}=\frac{1}{2}\left[\begin{array}{ccc}
0_{3} & I_{3} & 0_{3} \\
I_{3} & 0_{3} & 0_{3} \\
0_{3} & 0_{3} & 0_{3}
\end{array}\right]
$$

$A$ is a $(3 n \times 9)$ data matrix and $\mathbf{b}$ is a $(3 n \times 1)$ vector such that

$$
A=\left[\begin{array}{ccc}
\left(u_{1} s_{x}, v_{1} s_{y}, 1\right) & \otimes\left[{ }^{0} \mathbf{y}_{1}\right]_{\times} \\
\vdots & \\
\left(u_{n} s_{x}, v_{n} s_{y}, 1\right) & \otimes\left[{ }^{0} \mathbf{y}_{n}\right]_{\times}
\end{array}\right], \quad \mathbf{b}=\left[{ }^{0} \mathbf{w}_{1}{ }^{T}, \ldots,{ }^{0} \mathbf{w}_{n}{ }^{T}\right]^{T} .
$$

\subsection{Linear method with 4 points}

To obtain a minimal solution to the previous system (13), the $A$ matrix needs to be of rank 9 . From its construction, we know that each skew-symmetric matrix $\left[{ }^{0} \mathbf{y}_{i}\right]_{\times}$is of rank 2 . Thus, each correspondence potentially adds 2 independent rows to $A$ if the points are well distributed. The singularity of $A$ can be avoided by following the next geometric constraints during the selection of the image points:

1. Three fiducial lines of the object should not lie on the same plane, if it is the case, then the three projected points will be collinear in the image;

2. Three image points should not be collinear. This can accidentally happen even if the previous condition holds;

3. The vectors $\mathbf{y}_{i}$ should be different to ensure $A$ to be of sufficient rank. This also means that the lines should not be parallel in space.

With only 4 correspondences, the system (13) is always of deficient rank. Except for the special case arising when previous conditions are not respected, the matrix $A$ is of rank 8 , but its size is $12 \times 9$. It is still possible to build a family of solutions using the singular values decomposition (SVD), as proposed by Hartley \& Zisserman ${ }^{10}$ in computer vision theory. This one parameter solution will be fixed using the quadratic constraint (i.e. ${ }^{0} \mathbf{r}_{1}{ }^{T}{ }^{0} \mathbf{r}_{2}=$ 0 ). The following method is proposed

1. Compute the SVD of $A$ : $A=U D V^{T}$, with diagonal values $d_{i}$ from $D$ being sorted in decreasing order;

2. Compute $\mathbf{b}^{\prime}=U^{T} \mathbf{b}$,

3. Build a vector $\mathbf{z}$ such that $z_{i}=b_{i}^{\prime} / d_{i}$, for $i=1, \cdots, 8$ and $z_{9}=0$, 
4. The general one-parameter solution is $\mathbf{x}=S_{9}^{-1} V \mathbf{z}+\gamma S_{9}^{-1} \mathbf{v}_{9}$, with $\mathbf{v}_{9}$ the last column of $V$ and

$$
S_{9}^{-1}=\left[\begin{array}{ccc}
{ }^{\pi} S_{I}^{T} & 0 \\
0 & 0 & 1
\end{array}\right]^{-1} \otimes I_{3}
$$

5. Solve the scalar $\gamma$ using the quadratic constraints and finally compute the pose parameters.

The scalar $\gamma$ is computed by considering the family of solutions $\mathbf{x}=S_{9}^{-1} V \mathbf{z}+\gamma S_{9}^{-1} \mathbf{v}_{9}$. Considering only the first 6 components of $\mathbf{x}$, the first two columns of ${ }^{0} R_{c t}$ are written as affine functions of $\gamma$ :

$$
{ }^{0} \mathbf{r}_{1}=\mathbf{a}_{1}+\gamma \mathbf{b}_{1}, \quad{ }^{0} \mathbf{r}_{2}=\mathbf{a}_{2}+\gamma \mathbf{b}_{2}
$$

And the quadratic constraints are such that:

$$
\left\|{ }^{0} \mathbf{r}_{1}\right\|=1, \quad\left\|{ }^{0} \mathbf{r}_{2}\right\|=1, \quad{ }^{0} \mathbf{r}_{1}{ }^{T}{ }^{0} \mathbf{r}_{2}=0 .
$$

From one of the above equations, one can compute two scalar values for $\gamma$, for which only one solution is valid $\left(\left|\mathbf{x}_{(i)}\right| \leq 1\right.$, for $\left.i=1, \cdots, 6\right)$. In case of noise corruption and numerical errors, ${ }^{0} R_{c t}$ is generally not orthonormal. To enforce this property, a third SVD decomposition can be employed such that ${ }^{0} R_{c t}=U \Sigma V^{T}$. Then, the orthonormality of ${ }^{0} R_{c t}$ can be forced by choosing $\Sigma^{\prime}=I_{3}$ instead of $\Sigma$. The corrected rotation matrix is finally ${ }^{0} R_{c t}{ }^{\prime}=U \Sigma^{\prime} V^{T}\left({ }^{11,12}\right)$.

\subsection{Linear method with $N$ points}

With more than 4 correspondences, the matrix $A$ is of rank 9 (provided that the geometric constraints are respected). Hence the previous algorithm does not provide a correct answer to the pose reconstruction problem. In Eq. (12), the pose can be expressed as the solution minimizing the problem with a least-squares sense. This solution is found in closed-form using the classical formula $A^{T} A \mathbf{x}=A^{T} \mathbf{b}$. According to the knowledge of the authors, there is no other way to introduce the orthogonality constraint a priori in a generalized eigenvalue problem to get a unique solution for $\mathbf{x}$. The solution the author propose is to orthonormalize ${ }^{0} R_{c t}$ using a SVD decomposition as proposed in the previous algorithm.

It has to be pointed out that the accuracy increases with the addition of other correspondences provided that noise on image point locations is of Gaussian behavior. However, if an outlier is detected instead of a projection of an object line, a bias in the estimation may be significant and distributed on all the estimated parameters. In the next section, we will show how to deal with the presence of outliers among the set of image points.

\section{AUTOMATIC REGISTRATION PROCESS}

\subsection{Image processing}

A first image processing step is done by segmenting the CT-image (DICOM format) with a proper threshold level (+2000 Hounsfield units). All the centroids of the segmented spots are placed in a list (possible spots candidates) and another list contains the fiducial lines in Plückerian representation.

\subsection{Matching process}

The simultaneous pose reconstruction and matching problem is well known in computer vision. To achieve a robust estimation, we use a method that consists in testing an hypothesis of a pose obtained from a small group of correspondences. If this hypothesis gives good results for the other unmatched points (spots close to projected points), then the pose is validated. ${ }^{13}$ Often, $n_{o}$ of object lines have to match a large number $n_{I}$ of image points ( $n_{o} \neq n_{I}$ in case of outliers or inliers missing). The naïve method to find the best pose reconstruction is to try each possible permutation of four detected spots among the list of the spots with four elements of the fiducial list (generating the set of hypotheses). The validation is then ensured by testing the error made on the predicted position of the others spots with the remaining image points. This algorithm is slow since a lot of outliers can be detected in the segmentation, that in turns create a lot of rejected poses. 
Our algorithm is based on a $\mathrm{RANSAC}^{10,14}$ approach to have a fast and reliable automatic matching process. To find the best transformation between the $n_{I}$ points and $n_{o}$ object lines, a minimum subset of $N$ samples of 4 image points is selected among the full set et tested against every arrangements of 4 object lines. The number of pose reconstructions to be computed is $N n_{o}$. For each reconstruction, the number of matched points is stored. A point is considered to match an object line if the projection of the line is close to the point. The best pose is finally selected as the one that gives the maximum number of matches and that give a minimum error in the image.

\section{RESULTS}

By installing the robot with a needle on the translating table of the scanner, we propose to look at the error made in a point and click motion made by the robot after it is registered in the CT-image.

\subsection{Experimental setup}

Once an insertion point and a target is chosen in the CT-image, the CT-Bot will have to do the automatic positioning with respect to the selected trajectory (entry point, target to reach). For that, the device is registered in the CT-images in order to compute the relative transformation between the image plane and the end-effector holding the needle. The stereotactic fiducial objects used are made of $30 \times 30 \times 40 \mathrm{~mm}$ plastic cubes with six metal $\operatorname{rods}^{8}$ of $1 \mathrm{~mm}$ in diameter. These cubes are placed on the base of the robot to estimate the position and orientation of the end effector.

\subsection{Automatic pose reconstruction}

Without human intervention, it is possible to estimate the position of the robotic device holding the needle with an accuracy smaller than $1 \mathrm{~mm}$ in position with $512 \times 512$ image with $0.5 \mathrm{~mm}$ scaling factors. The processing time depends on the number of outliers that are in the list of the spots. In common cases (6 inliers and 2 outliers), the registration time is about $100 \mathrm{~ms}$ with a Pentium 4 computer at $2.4 \mathrm{Ghz}$, which is acceptable even for real-time processing.

The registration process gives a mean error of 0.23 pixel on the six fiducial rods in a serie of 10 acquisitions and registrations. That means that the mean distance between the spots and the reconstructed positions with the estimated pose is less than $0.12 \mathrm{~mm}$. Hence, a pixel point in the image is mapped to a spatial position with a good accuracy, provided this point is not too far from the fiducial object.

\subsection{Needle Positioning with a reference point in the image}

Using the graphical user interface, we indicate on the screen the current tip position of the needle and the position of the target to reach (another metal needle). This translation is mapped to a relative Cartesian displacement to execute by the robotic device. In our different tests, the translation was mapped to a vector which coordinates are not null on the different axis $(\mathrm{X}, \mathrm{Y}, \mathrm{Z})$. A common translation was $[25,3,-7]^{T} \mathrm{~mm}$. With this displacement, the needle should be visible in the target image, close to the reference point. A result can be seen on the Fig. 4 and Fig. 5 where one can easily check the position of the tip.

The trials of visual positioning have been successfully done with a final error that is less than $1 \mathrm{~mm}$ (2 pixels in the image). The measure is difficult to quantify since we cannot mechanically measure the position of the tip of the needle with an external device.

\section{DISCUSSION}

Firstly, the robot calibration error is inherent to the mechanism. This error should be considered as a constant error that does not influence the relative Cartesian displacement. This is important when absolute positioning is required. Then, the error in the single slice pose reconstruction directly affects the visual positioning. It should be noted that the sensibility of the global needle registration method is mainly correlated with the pose reconstruction which is done with an average error smaller than $1 \mathrm{~mm}$.

The current entry point selection and tip detection is based on pixel information given by mouse clicks. This information is to consider with caution since the accuracy in mainly the responsibility of the operator. 

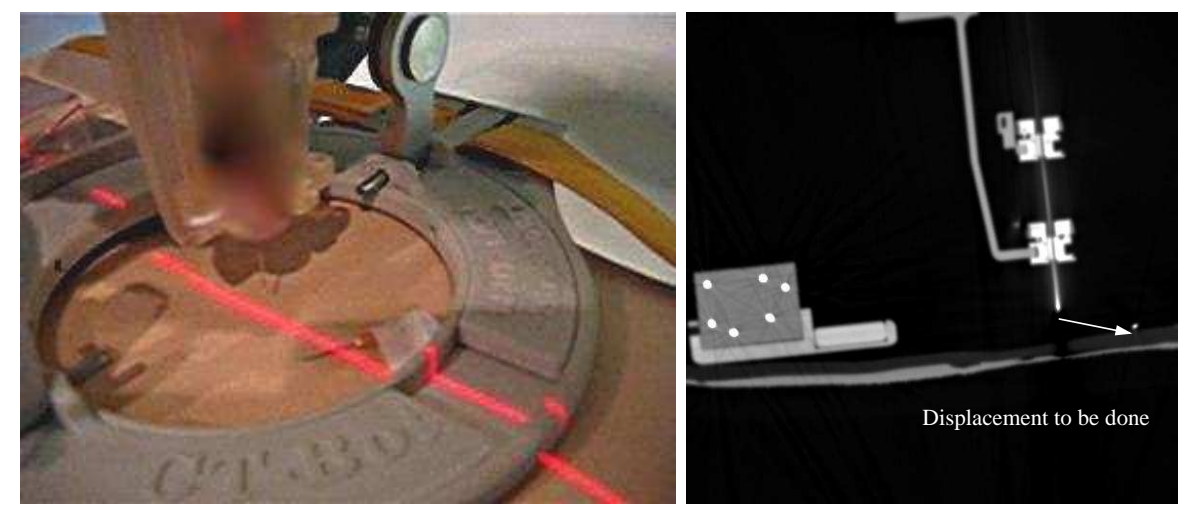

Figure 4. Needle position before the registration has been done. Note the visibility of the fiducial cube in the CT-image.
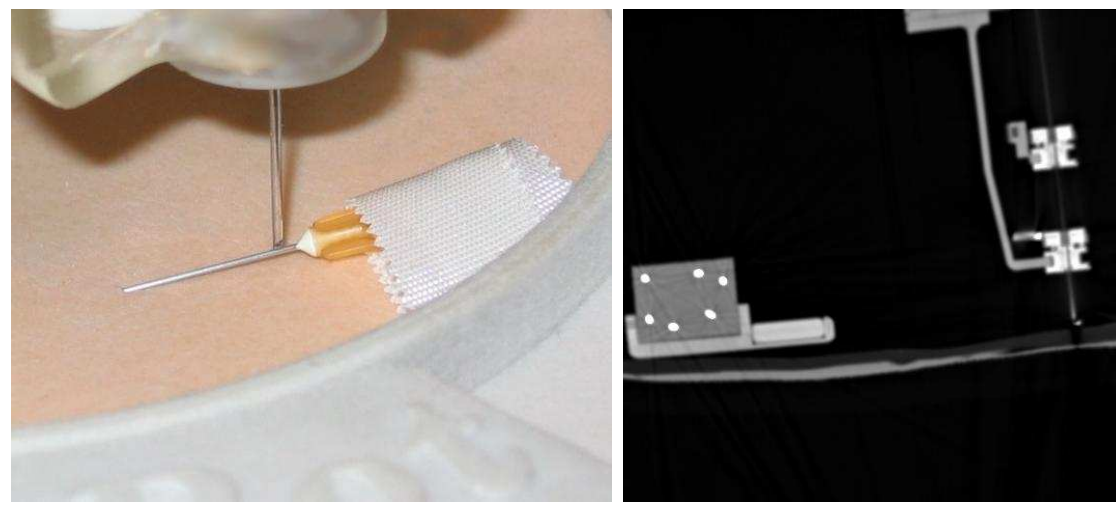

Figure 5. Needle position after the pointing has been done. In the resulting CT-scan image, an error lower than $1 \mathrm{~mm}$ is achieved.

\section{CONCLUSION}

This paper presents registration tests made using a safe robotic assistant for percutaneous procedures on the abdomen with a computed tomography imaging device. To help radiologists during the entry point selection, a critical part of the insertion of a needle, we propose to give an image-based robotic assistance. A short description of the experimental setup is given and the stereotactic registration process is described. With this technique, we achieve a good registration accuracy of the robotic system. The results of point-to-point motions of the tip of a needle with an image reference is very promising for the futur. This image-to-robot registration will be necessary for further automatic visual guidance.

\section{ACKNOWLEDGMENTS}

The authors wish to thank the Alsace Region Council and the French CNRS Robea project for the financial support of this research project.

\section{REFERENCES}

1. R. H. Taylor, "Medical robotics and computer aided surgery," September 2002. Web PDF Hands-out of presentation at MICCAI'02.

2. B. Maurin, J. Gangloff, B. Bayle, M. de Mathelin, O. Piccin, P. Zanne, C. Doignon, L. Soler, and A. Gangi, "A parallel robotic system with force sensors for percutaneous procedures under CT-guidance," in Proceedings of the 2004 Medical Image Computing and Computer-Assisted Intervention Conference (in press), MICCAI'04, (St-Malo, France), September 2004. 
3. B. Maurin, O. Piccin, B. Bayle, J. Gangloff, and M. de Mathelin, "A parallel 5 dof positioner for semispherical workspaces," in Proceedings of the 2004 ASME Design Engineering Technical Conferences (in press), DETC'04, (Salt Lake City, USA), October 2004.

4. K. Cleary, M. Freedman, M. Clifford, D. Lindisch, S. Onda, and L. Jiang, "Image-guided robotic delivery system for precise placement of therapeutic agents," Journal of Controlled Release 74, pp. 363-368, 2001.

5. G. Fichtinger, T. L. DeWeese, A. Patriciu, A. Tanacs, D. Mazilu, J. H. Anderson, K. Masamume, R. H. Taylor, and D. Stoianovici, "Robotically assisted prostate biopsy and therapy with intra-operative CT guidance," Journal of Academic Radiology 9, pp. 60-74, 2002.

6. R. C. Susil, J. H. Anderson, and R. H. Taylor, "A single image registration method for ct guided interventions," in Proceedings of the International Conference on Medical Image Computing and Computer-Assisted Intervention (MICCAI 99), pp. 798-808, 1999.

7. S. Lee, G. Fichtinger, and G. S. Chirikjian, "Numerical algorithms for spatial registration of line fiducials from cross-sectional images," American Association of Physicists in Medicine 29, pp. 1881-1891, Aug. 2002.

8. B. Maurin, C. Doignon, M. de Mathelin, and A. Gangi, "Pose reconstruction with an uncalibrated computed tomography imaging device," in Proceedings of the IEEE International Conference on Computer Vision and Pattern Recognition, 1, pp. 455-460, CVPR 2003, June 2003.

9. R. A. Brown, T. S. Roberts, and A. G. Osborne, "Stereotactic frame and computer software for CT-directed neurosurgical localization," Invest. Radiol. 15, pp. 308-312, 1980.

10. R. Hartley and A. Zisserman, Multiple view geometry in computer vision, Cambridge University Press, 2000.

11. S. Umeyama, "Least-squares estimation of transformation parameters between two point patterns," IEEE Transactions on Pattern Analysis and Machine Intelligence 13, pp. 376-380, April 1991.

12. D. Goryn and S. Hein, "On the estimation of rigid body rotation from noisy data," IEEE Transactions on Pattern Analysis and Machine Intelligence 17, pp. 1219-1220, Dec. 1995.

13. E. Grimson, Object Recognition by Computer: The Role of Geometric Constraint, MIT Press, 1990.

14. M. A. Fischler and R. C. Bolles, "Random sample consensus: A paragim for model fitting with applications to image analysis and automated cartography," Communications of the ACM 24(6), pp. 381-395, 1981. 